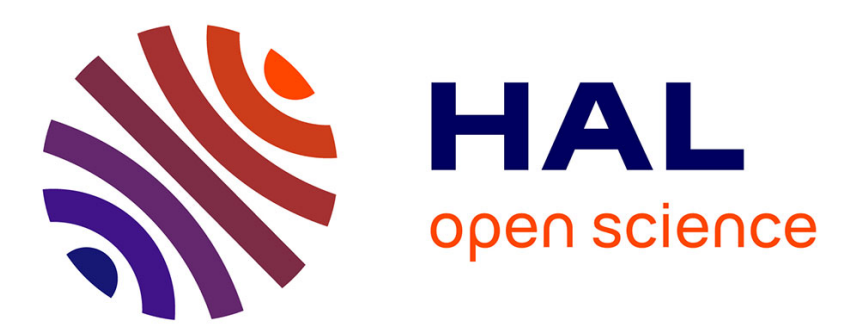

\title{
Single pixel camera: an acquisition strategy based on the non-linear wavelet approximation
}

Florian Rousset, Nicolas Ducros, Cosimo d'Andrea, Françoise Peyrin

\section{To cite this version:}

Florian Rousset, Nicolas Ducros, Cosimo d'Andrea, Françoise Peyrin. Single pixel camera: an acquisition strategy based on the non-linear wavelet approximation. EMBC 2015, Aug 2015, Milan, Italy. hal-01217883

\section{HAL Id: hal-01217883 \\ https://hal.science/hal-01217883}

Submitted on 20 Oct 2015

HAL is a multi-disciplinary open access archive for the deposit and dissemination of scientific research documents, whether they are published or not. The documents may come from teaching and research institutions in France or abroad, or from public or private research centers.
L'archive ouverte pluridisciplinaire HAL, est destinée au dépôt et à la diffusion de documents scientifiques de niveau recherche, publiés ou non, émanant des établissements d'enseignement et de recherche français ou étrangers, des laboratoires publics ou privés. 


\title{
Single pixel camera: an acquisition strategy based on the non-linear wavelet approximation
}

\author{
Florian Rousset ${ }^{1}$, Nicolas Ducros ${ }^{1}$, Cosimo D'Andrea ${ }^{2}$ and Françoise Peyrin ${ }^{1}$
}

\begin{abstract}
Single pixel imaging opened the door to a cheaper camera architecture able to operate in a wide spectral range. Compressive sensing has been used with such an optical setup to reconstruct an image using $\ell_{1}$-minimization. To avoid this type of reconstruction, we consider an adaptive approach leading to a direct restoration of an image and for which we propose a new acquisition strategy. Our technique allows one to acquire an image in the wavelet domain with a progressive non-linear acquisition strategy. This scheme is based on the non-linear approximation of the wavelet transform which takes advantage of the transformation's sparsity. This approximation is applied in a multiresolution way and is shown to offer high compression performance on simulated data. One application of the single pixel camera concerns time-resolved acquisition to observe fluorescence lifetime images of biological structures.
\end{abstract}

\section{INTRODUCTION}

The single pixel camera (SPC) architecture is the key to building small, cheap and efficient sensors. Compared to CCD or CMOS cameras architecture, SPC has several advantages. This imaging technique can indeed operate at different wavelengths where building CCD or CMOS can be expensive. Infrared or multispectral imaging can therefore be considered. In addition, the single detector can have a very good quantum efficiency and few storage memory is needed compared to conventional imaging techniques. Having only one detector, SPC is also well suited for timeresolved acquisition using a single TCSPC (Time-Correlated Single Photon Counting) board.

Our goal in this paper is to provide a new acquisition strategy for SPC acquisitions that leads to a low cost time-resolved imaging technique. One application of this method could be the observation of biological tissues via fluorescence imaging. In particular, the overall framework could benefit to optical tomography for preclinical imaging of animals [1]. Fluorescence lifetime imaging can also be considered using the SPC as a low cost time-resolved acquisition device [2].

\section{PROBLEM AND RELATED WORK}

We address the problem of recovering the image of an object acquired by a SPC, which was originally formulated in [3], [4]. The optical setup consists of a digital micromirror device (DMD) and a single detector element (e.g. single photon avalanche diode or photomultiplier). A lens is added

${ }^{1}$ F. Rousset, N. Ducros and F. Peyrin are with CREATIS, CNRS UMR5220, INSERM U1044, Université de Lyon, INSA Lyon, Bat Blaise Pascal, 69621 Villeurbanne Cedex, France. Corresponding author: florian.roussetecreatis.insa-lyon.fr

${ }^{2}$ C. D'Andrea is with the Department of Physics, Politecnico di Milano, Piazza Leonardo Da Vinci 32, Milano, Italy.

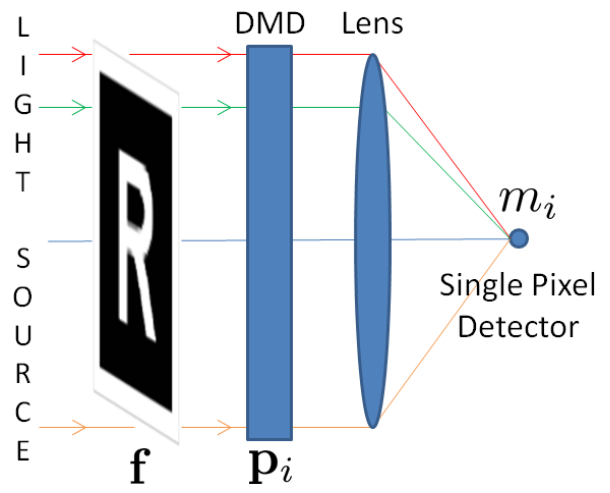

Fig. 1. Optical setup composed of a DMD and a single pixel detector. The measure at the detector corresponds to the projection of the image on a certain pattern.

to focus the light rays on the single detector as depicted in Fig. 1. A DMD is composed of thousands of mirrors that can be independently tilted in two states. The first one reflects the light toward the detector and is said to be the ON state. The second one, the OFF state, reflects the light in the opposite direction. Hence, a DMD can act as a tunable spatial filtering device.

A SPC acquisition consists in computing sequentially the dot product of the image and some DMD patterns. Let $\mathbf{f} \in \mathbb{R}^{N \times N}$ be the $N \times N$ image and $\left\{\mathbf{p}_{i} \in \mathbb{R}^{N \times N}, i=1 . . I\right\}$, a sequence of $I$ DMD patterns. The measurements $\left\{m_{i}, i=\right.$ $1 . . I\}$ can be expressed as

$$
m_{i}=\left\langle\mathbf{f}, \mathbf{p}_{i}\right\rangle
$$

Then, the problem consists in retrieving $\mathbf{f}$ from $\left\{m_{i}\right\}$, knowing the patterns $\left\{\mathbf{p}_{i}\right\}$.

In [3], [4], the authors used compressed sensing [5]. This approach is nonadaptive in the sense that random patterns are considered, their elements being drawn as independent and identically distributed \pm 1 random variables from a uniform Bernoulli distribution. The image $\mathbf{f}$ is then restored with $\ell_{1}$ minimization. In [6], [7], [8], the image is restored directly, i.e.

$$
\mathbf{f}=\sum_{i} m_{i} \mathbf{p}_{i}
$$

and the image was acquired with an adaptive scheme. Some of the patterns were determined during acquisition depending on measurements already acquired. The difficulty lies not so much in recovering the image as in determining the DMD patterns. 
In this paper, we consider the same type of approach since it avoids the time-consuming $\ell_{1}$-minimization. In particular, we consider to obtain $\left\{m_{i}\right\}$ from wavelet patterns $\left\{\mathbf{p}_{i}\right\}$ using a non-linear acquisition strategy.

\section{METHODS}

\section{A. Wavelet decomposition}

The discrete wavelet decomposition of an image $\mathbf{f} \in \mathbb{R}^{N \times N}$ with the standard dyadic wavelets separates the signal into approximation and detail coefficients (horizontal, vertical or diagonal). The approximation coefficients result from a low-pass filtering, detail coefficients from a high-pass filtering [9].

Let $j=1 . . J$ be the scale at which the image $\mathbf{f}$ is observed, $J$ being the decomposition level of the wavelet transform, with $1 \leq J \leq \log _{2} N=R$ and $k=\left(k_{1}, k_{2}\right) \in \mathbb{Z}^{2}$ specify a location. Let $\beta_{J, k}$ be the approximation coefficients and $\gamma_{j, k}^{e}$ the detail coefficients with $e=1,2$, or 3 representing the horizontal, vertical and diagonal coefficients, respectively. These elements can be obtained by:

$$
\beta_{J, k}=\left\langle\mathbf{f}, \phi_{J, k}\right\rangle \quad \gamma_{j, k}^{e}=\left\langle\mathbf{f}, \psi_{j, k}^{e}\right\rangle
$$

where $\phi$ and $\psi$ are respectively the scaling and wavelet basis functions. Equations (1) and (2) show that each wavelet coefficient can be computed by the SPC using its scaling and wavelet functions as detection patterns. In an orthonormal basis, one can recover an image from its wavelet coefficients using the inverse wavelet transform given by:

$$
\mathbf{f}=\sum_{k \in \mathbb{Z}^{2}} \beta_{J, k} \phi_{J, k}+\sum_{e=1}^{3} \sum_{j=1}^{J} \sum_{k \in \mathbb{Z}^{2}} \gamma_{j, k}^{e} \psi_{j, k}^{e}
$$

Wavelet decomposition was shown to give sparse signals, allowing one to discard many of the coefficients at the reconstruction step [9]. This sparsity is exploited by compression algorithms such as JPEG2000 [10].

\section{B. Patterns loaded on the DMD}

While wavelet patterns generally have both negative and positive entries, the DMD only allows positive patterns to be uploaded. Given the linearity of (1), a solution consists in splitting a pattern $\mathbf{p}$ into its positive and negative parts, $\mathbf{p}^{+}$ and $\mathbf{p}^{-}$, respectively, such that $\mathbf{p}=\mathbf{p}^{+}-\mathbf{p}^{-}$. Henceforth, the corresponding measure is easily obtained as the difference of two measures:

$$
m=m^{+}-m^{-}
$$

where

$$
m^{+}=\left\langle\mathbf{f}, \mathbf{p}^{+}\right\rangle \quad \text { and } \quad m^{-}=\left\langle\mathbf{f}, \mathbf{p}^{-}\right\rangle
$$

Haar's wavelet is considered in this paper since its separated patterns only take two values. Using a scale factor, each pattern can be transformed to patterns with only 0 or 1 values. This is well suited for the ON/OFF technology of the DMD.

Moreover, today's DMDs can produce up to 1024 gray levels and should allow the use of other wavelets with better compression skills.

\section{Acquisition strategy}

Given the sparsity of the wavelet transform, acquiring each coefficient would be a time loss. Hence, a sampling scheme has to be chosen to mainly acquire significant coefficients. Deutsch et al. [6] considered a father-son relationship based on the tree structure of the wavelet decomposition [9]. It stands that a coefficient at the scale $j$ has 4 sons at the scale $j-1$. Then, they employed a thresholding strategy to predict the relevant coefficients at finer and finer scales. The fatherson relationship is used in their strategy given that wavelet coefficients tend to persist through scale, i.e. a significant coefficient at the scale $j$ may have 4 significant sons at $j-1$. Dai et al. [7] considered the same type of approach but with a more refined thresholding strategy that performs better than the previous method. To overcome the limits of an imagedependant thresholding strategy, we propose instead to nonlinearly determine the relevant coefficients.

We base our method on the non-linear approximation of the wavelet transform. This technique retains a number $M<<N^{2}$ of the largest wavelet coefficients and was shown to give excellent image recovery [9]. However, having no knowledge of the full wavelet transform of the object, we perform several non-linear approximations throughout the decomposition levels. Our strategy therefore consists in the following steps. First, we acquire the approximation of the image at scale $J$, which provides $n_{\mathrm{A}}=2^{2 L}$ coefficients with $L=R-J$. Then, the approximation image is one-level wavelet transformed and a given percentage $p_{J}$ of the largest detail coefficients are retained. The four sons of each of the retained detail coefficients are chosen for acquisition. The number $n_{J}$ of detail coefficients acquired at scale $J$ is given by

$$
n_{J}=3 \times 2^{2(L-1)} \times 4 \times p_{J}=3 \times n_{\mathrm{A}} \times p_{J} .
$$

Then, we perform another non-linear approximation among the $n_{J}$ details by keeping a new percentage $p_{J-1}$ of the largest ones. As previously, the four children of the significant details will be considered for measurement. The number $n_{J-1}$ of measured detail coefficients at $J-1$ is

$$
n_{J-1}=4 \times p_{J-1} \times n_{J}
$$

Repeating the previous step until the finest scale $j=1$ is reached, one can obtain the total number of acquired coefficients $n$ with:

$$
n=2^{2 L}\left[1+3 \sum_{j=1}^{J}\left(4^{J-j} \prod_{i=j}^{J} p_{i}\right)\right]
$$

The set of percentages $\mathscr{P}=\left\{p_{1}, p_{2}, \ldots, p_{J}\right\}$ controls the number of sampled coefficients over the total number of pixels (6). The latter ratio defines the sampling rate (SR). One can finally recover an image from the samples using the inverse wavelet transform (3).

\section{RESULTS}

The proposed acquisition strategy was applied to several test images and compared to the global non-linear approximation (GNLA) as well as to Dai's acquisition strategy. The 

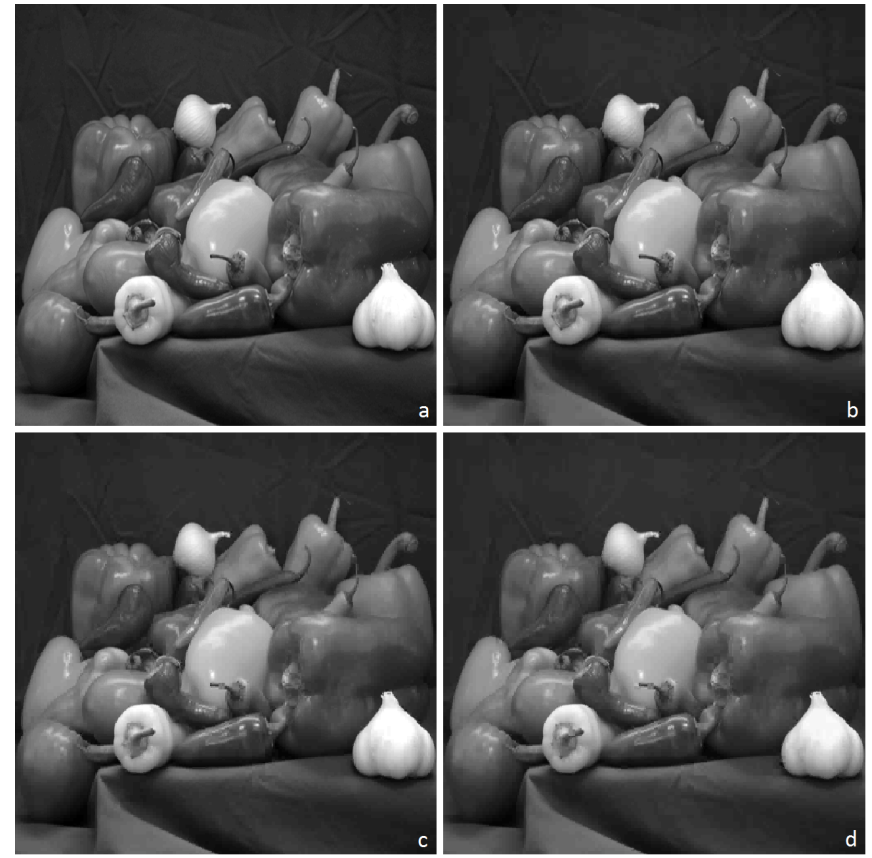

Fig. 2. Results of different methods on a $512 \times 512$ pixels image. (a) Ground truth image. (b) Recovered image using a GNLA with a SR of 10 $\%$ giving a PSNR of $41.29 \mathrm{~dB}$. (c) Recovered image using our technique with a SR of $10 \%$ giving a PSNR of $36.07 \mathrm{~dB}$. (d) Recovered image using Dai's method with a SR of $10 \%$ giving a PSNR of $35.76 \mathrm{~dB}$.

GNLA consists in retaining the largest $M<<N^{2}$ wavelet coefficients on the whole wavelet transform [9]. Dai et al. [7] recently proposed approach is based on Haar's properties, the father-son relationship and a thresholding acquisition strategy.

In Fig. 2, we first show results obtained on a single image for different strategies. In each case, the sampling rate was fixed to $10 \%$.

Figure 3 highlights the sampled wavelet coefficients in our strategies compared to the GNLA.

Table II presents the PSNRs obtained for different standard test images considering both our proposed and Dai's technique, showing that the proposed strategy seems to perform better.

Fluorescence imaging being a target application, we tested our acquisition strategy on an image of a mouse injected with a fluorescence dye, for different SRs, as reported in Fig. 4 and table I. Even with a SR as low as $2 \%$, our method shows efficient results with most of the structures still visible in the recovered image.

\section{DISCUSSION}

\section{A. Comparison with a global non-linear approximation}

The global non-linear approximation gives the best result with a PSNR of $41.29 \mathrm{~dB}$ (Fig. 2). This comes from the approximation being applied to the whole wavelet transform that is in practice not applicable with the SPC. Our method, possible with the SPC, gives a PSNR of $36.07 \mathrm{~dB}$. This indicates that our strategy misses some of the significant wavelet coefficients found with the GNLA (Fig. 3). We

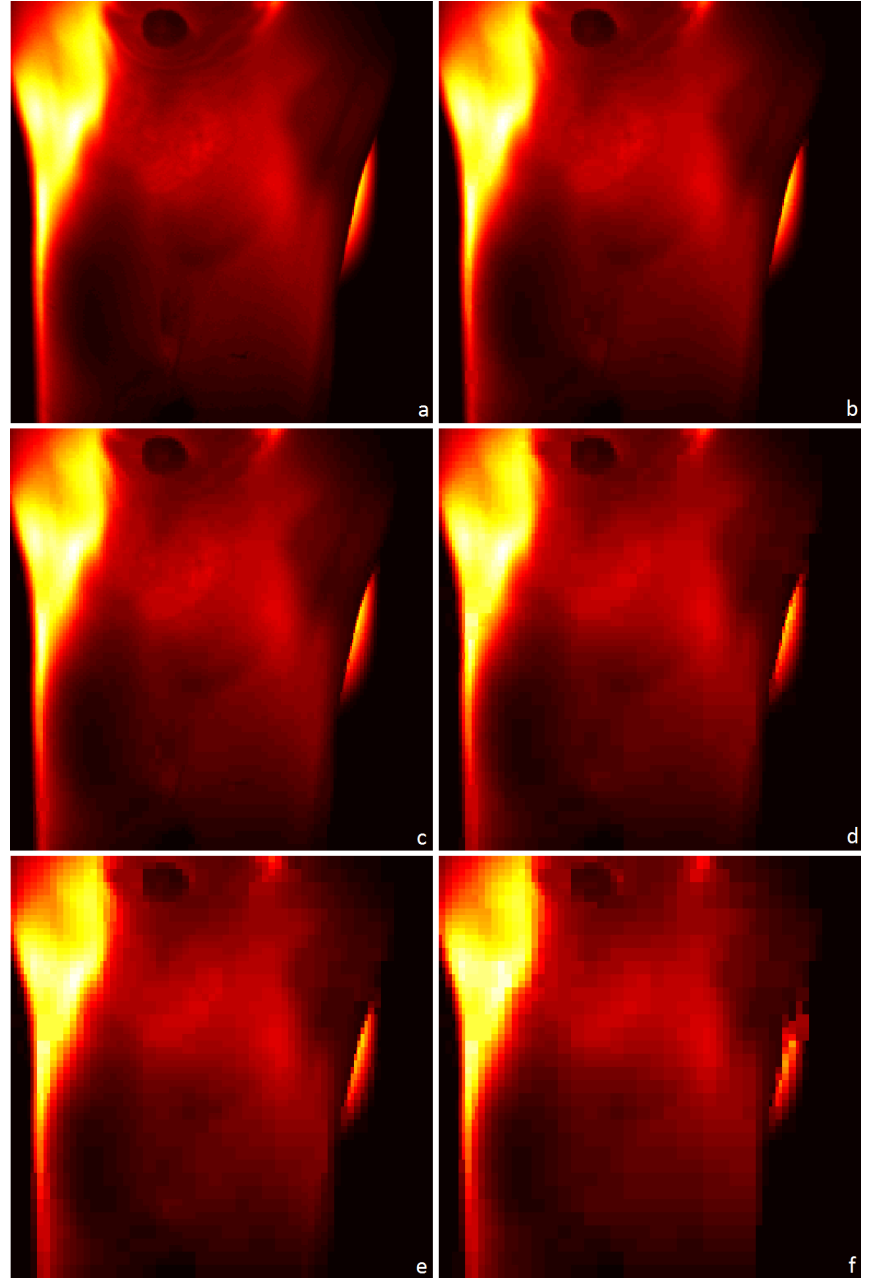

Fig. 4. Results of our approach on a fluorescence image $(256 \times 256)$ with different sampling rates. (a) Ground truth image, (b) images recovered with a SR of $15 \%$, (c) $10 \%$, (d) $5 \%$, (e) $3 \%$ and (f) $2 \%$. Table I presents the set of percentages and PSNR associated with these results.

can see that most of the significant coefficients are well found with our method for the coarser decomposition levels. However, at the finest decomposition level $j=1$, a lot of the significant coefficients are forgotten, restoring less details and inducing an important PSNR loss.

To get as close as possible to the GNLA, further researches based on super resolution methods [11] should allow us to predict with more accuracy the significant coefficients.

\section{B. Comparison with Dai's method}

In addition to better PSNRs (table II), our method presents adaptivity in the sense that an unique set of percentages works well for several images whereas a threshold is imagedependent. This can be seen by comparing the cases (c) of Fig. 2 and Fig. 4. For both cases, the same set of percentages was used. In the fluorescence case, the image have clearly smooth areas whereas the considered image in Fig. 2 have sharp edges. Despite this difference, the recovered images are very close to their ground truth versions, showing the powerfulness and adaptivity of our method. 

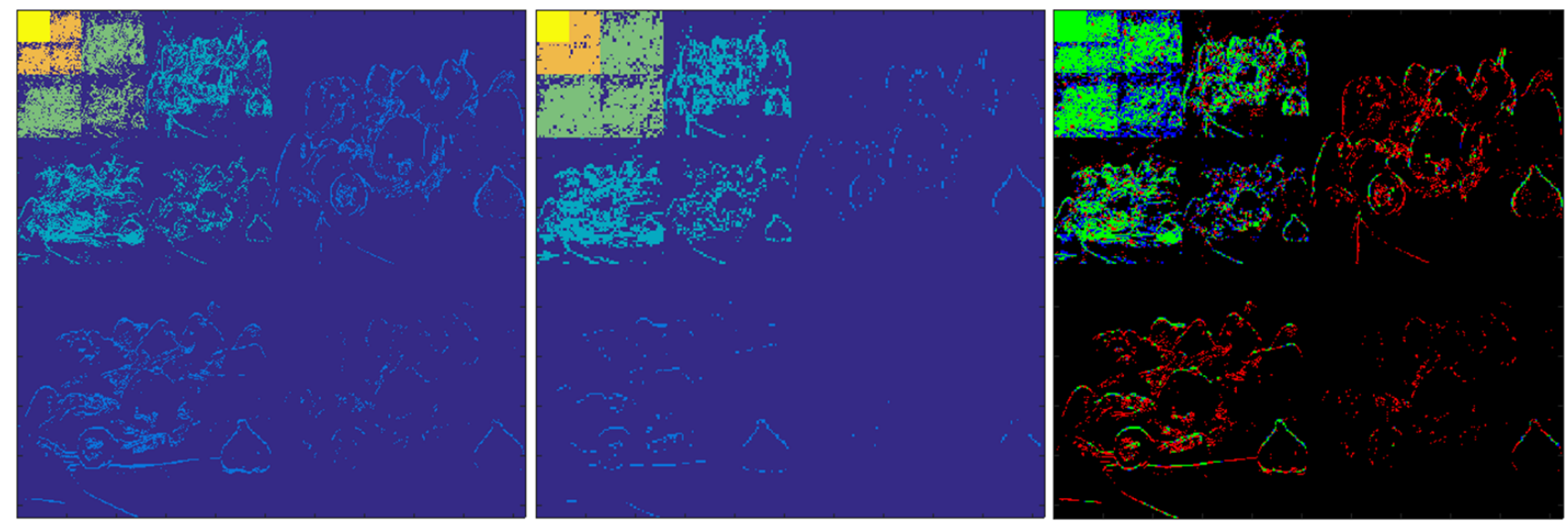

Fig. 3. Comparison of the calculated coefficients for a SR of $10 \%$ between our method and a GNLA in the case of Fig. 2. Left: sampled coefficients with a GNLA (1). Middle: sampled coefficients using our method (2). Right: comparison showing the coefficient sampled in both method (in green), sampled with (1) but not (2) (in red), sampled with (2) but not (1) (in blue).

\begin{tabular}{cccc}
\hline \hline Case & Sampling rate & Set of percentages & PSNR (dB) \\
\hline (b) & $15 \%$ & $\{0.1,0.45,0.85,0.95\}$ & 46.31 \\
(c) & $10 \%$ & $\{0.05,0.3,0.8,0.95\}$ & 43.50 \\
(d) & $5 \%$ & $\{0.01,0.2,0.5,0.85\}$ & 38.15 \\
(e) & $3 \%$ & $\{0.01,0.05,0.4,0.8\}$ & 35.97 \\
(f) & $2 \%$ & $\{0,0.04,0.3,0.6\}$ & 33.20 \\
\hline \hline
\end{tabular}

TABLE I

SET OF PERCENTAGES AND PEAK SIGNAL TO NOISE RATIOS ASSOCIATED WITH THE RESULTS OF FIG. 4.

\begin{tabular}{ccc}
\hline \hline Test & PSNR (dB) & PSNR (dB) \\
Non-linear & Threshold \\
\hline Lena $(512 \times 512)$ & 30.33 & 29.84 \\
Peppers $(512 \times 512)$ & 36.07 & 35.76 \\
Cameraman $(512 \times 512)$ & 32.32 & 32.05 \\
Gold Hill $(512 \times 512)$ & 27.83 & 27.70 \\
\hline \hline
\end{tabular}

TABLE II

PSNR BETWEEN GROUND TRUTH IMAGES AND THEIR RECOVERED VERSIONS. $\mathscr{P}=\{0.05,0.3,0.8,0.95\}$ WAS USED FOR OUR NON-LINEAR STRATEGY (COLUMN 2) AND FOR DAI'S METHOD (COLUMN 3), THE THRESHOLD WAS CHOSEN BASED ON THE APPROXIMATION IMAGE [7]. IN BOTH CASES, THE SR WAS FIXED TO $10 \%$.

Moreover, the set of percentages $\mathscr{P}$ also allows us to control the ratio of sampled coefficients in each decomposition level where it is impossible with the thresholding strategy. Finally, any wavelet (orthogonal and biorthogonal) can be used with our technique, the only limit being the DMD's positivity restriction.

\section{CONCLUSION}

We presented in this paper a strategy to acquire images with a SPC. Using an adaptive approach, we avoid the computation overhead of compressed imaging to reconstruct an image via $\ell_{1}$-minimization. Instead, we obtain an image in the wavelet domain using a progressive non-linear approximation strategy. This allows us to directly restore an image with the inverse wavelet transform. Simulation tests of the proposed methodology show both good visual and numerical results. In future work, we plan to use this optical setup for fluorescence lifetime imaging of biological tissues. In such imaging, SPC allows for a low cost time-resolved imaging device using only one TCSPC board.

\section{REFERENCES}

[1] Nicolas Ducros, Andrea Bassi, Gianluca Valentini, Gianfranco Canti, Simon Arridge, and Cosimo D'Andrea. Fluorescence molecular tomography of an animal model using structured light rotating view acquisition. Journal of Biomedical Optics, 18(2):020503-020503, 2013

[2] Nicolas Ducros, Anabela Da Silva, Jean-Marc Dinten, Chandra Sekhar Seelamantula, Michael Unser, and Françoise Peyrin. A time-domain wavelet-based approach for fluorescence diffuse optical tomography. Medical Physics, 37(6):2890-2900, 2010.

[3] Dharmpal Takhar, Jason N. Laska, Michael B. Wakin, Marco F. Duarte, Dror Baron, Shriram Sarvotham, Kevin F. Kelly, and Richard G. Baraniuk. A new compressive imaging camera architecture using optical-domain compression. In in Proc. of Computational Imaging IV at SPIE Electronic Imaging, pages 43-52, 2006.

[4] M.F. Duarte, M.A. Davenport, D. Takhar, J.N. Laska, Ting Sun, K.F. Kelly, and R.G. Baraniuk. Single-pixel imaging via compressive sampling. Signal Processing Magazine, IEEE, 25(2):83-91, March 2008

[5] David L. Donoho. Compressed sensing. IEEE Trans. Inform. Theory, 52:1289-1306, 2006.

[6] Shay Deutsch, Amir Averbush, and Shay Dekel. Adaptive compressed image sensing based on wavelet modeling and direct sampling. In Laurent Fesquet and Bruno Torrésani, editor, SAMPTA'09, page General session, Marseille, France, May 2009

[7] Huidong Dai, Guohua Gu, Weiji He, Fajian Liao, Jiayan Zhuang, Xingjiong Liu, and Qian Chen. Adaptive compressed sampling based on extended wavelet trees. Appl. Opt., 53(29):6619-6628, Oct 2014.

[8] A. Averbuch, S. Dekel, and S. Deutsch. Adaptive compressed image sensing using dictionaries. SIAM Journal on Imaging Sciences, 5(1):57-89, 2012

[9] Stephane Mallat. A Wavelet Tour of Signal Processing, Third Edition: The Sparse Way. Academic Press, 3rd edition, December 2008.

[10] D. S. Taubman and M. W. Marcellin. Jpeg2000: standard for interactive imaging. Proceedings of the IEEE, 90(8):1336-1357, 2002.

[11] Sung Cheol Park, Min Kyu Park, and Moon Gi Kang. Super-resolution image reconstruction: a technical overview. Signal Processing Magazine, IEEE, 20(3):21-36, 2003 